\title{
Homodyne laser radar system for surface displacement monitoring
}

\author{
Alejandro Rodriguez, MEMBER SPIE \\ Adolfo Comeron, MEMBER SPIE \\ David Garcia \\ Universitat Politècnica de Catalunya (UPC) \\ Electromagnetics and Photonics \\ Engineering Group \\ Departament de Teoria del Senyal i \\ Comunicacions \\ Campus Nord UPC, Edifici D-4 \\ C/Jordi Girona, 1-3 \\ 08034 Barcelona, Spain
}

\begin{abstract}
A prototype of a homodyne laser radar system for surface displacement monitoring using the reference beam technique is presented. The prototype is very simple, is easy to align and focus, and is able to measure the velocity of the surface displacement at distances up to $16 \mathrm{~m}$. We present an optical analysis of the prototype, a power budget, a criterion on tolerance in distance and laboratory measurements. (c) 2001 Society of Photo-Optical Instrumentation Engineers. [DOI: 10.1117/1.1347031]

Subject terms: laser radar; homodyne; optical mixing; velocity measurement; surface displacement measurement; HeNe laser; avalanche photodiode; Doppler shift; coherence.
\end{abstract}

Paper 200058 received Feb. 21, 2000; revised manuscript received Sep. 11, 2000; accepted for publication Sep. 25, 2000.

\section{Introduction}

Coherent laser radar systems can be used to monitor the speed of moving surfaces. Although systems based on a differential technique (namely, laser Doppler velocimeters ${ }^{1}$ ) can measure components of the velocity vector, they do not provide good performance figures at distances over a few centimeters.

Some homodyne systems have been proposed in the literature. Rudd ${ }^{2}$ presented a homodyne prototype that used a single HeNe laser source as transmitter, a local oscillator (reference beam) and a mixer, but this system (as shown formally by Potter $^{3}$ ) cannot measure Doppler shifts greater than $1 \mathrm{MHz}$ for targets located at distances farther than about $1 \mathrm{~m}$. Churnside proposed a similar system (meticulously studied in his papers ${ }^{4,5}$ ) based on a $\mathrm{CO}_{2}$ laser.

Some authors (see, for instance, Refs. 6 and 7) have reported systems based on laser diodes that perform the mixing on the same source. The results presented are promising, but the short coherence length of the light produced by actual laser diodes still limits their performance.

In this paper, we present a homodyne coherent laser radar system, based on a HeNe laser, that measures Doppler shifts up to $\sim 93 \mathrm{MHz}$ when a laboratory target is placed at distances ranging from 1 to $16 \mathrm{~m}$. In this system, the mixing takes place on the active surface of an avalanche photodiode (APD), so it does not have the limitations of the system proposed by Rudd. ${ }^{2}$ In Section 2 the prototype built is described and the theory of operation is explained. Section 3 is devoted to the optical analysis of the prototype. Section 4 presents a power budget and proposes a criterion concerning the tolerance in the distance adjustment of such a system. Section 5 describes some experimental results.

\section{Prototype Description and Theory of Operation}

The prototype developed is shown in Fig. 1 (Refs. 8 and 9). A Siemens LGK7627 HeNe laser is employed as the transmitter and local oscillator source and an Analog Modules 713-4 APD/transimpedance amplifier set is used as the pho- toreceiver. A homemade beamsplitter and two general purpose lenses (with focal lengths 1 and $15 \mathrm{~cm}$ ) are used as the transmitting/receiving/mixing $(\mathrm{Tx} / \mathrm{Rx} / \mathrm{Mx})$ optics. The components are fixed on an optical board.

The transmitted beam is expanded and then focused on the target, using the telescope formed by the two lenses. The distance $d_{3}$, where the beam waist lays, can be controlled by changing the separation $d_{1}$ between the laser and lens 1 , with the overall distance $D$ between the laser and lens 2 a constant. The target scatters some of the incident light, which has been frequency-shifted by the Doppler effect. The frequency shift can be written:

$\Delta f_{D}=2 \frac{v_{r}}{\lambda}$,

where $\lambda$ is the wavelength of the light produced by the laser $(633 \mathrm{~nm})$.

Part of the scattered light is collected by the Tx/Rx/Mx optics and directed to the laser output mirror, where it is reflected. Approximately 5\% of both the received signal and transmitted beam (which plays the role of the local oscillator, LO) powers are reflected by the beamsplitter and impinge on the APD active area. In this way, the interference between the LO beam and the received light can be detected and amplified. The resulting voltage $v_{\text {Dopp }}(t)$, called the Doppler signal from here on, has a dc component due to the average incident light power and an ac component due to the interference between the LO and the received light. It can be written as ${ }^{1}$

$v_{\text {Dopp }}(t)=\rho_{v} 2\left(\eta_{\text {het }} P_{\text {LO }} P_{\mathrm{Rx}}\right)^{1 / 2} \cos \left(2 \pi \Delta f_{D} t+\chi\right)$,

where $\rho_{v}$ is the voltage responsivity (in volts per watt) of the photoreceiver; $\eta_{\text {het }}$ is the so-called heterodyne efficiency, ${ }^{10,11} P_{\mathrm{LO}}$ and $P_{\mathrm{Rx}}$ are the local-oscillator and received-signal powers, respectively; and $\chi$ is an unknown phase. 


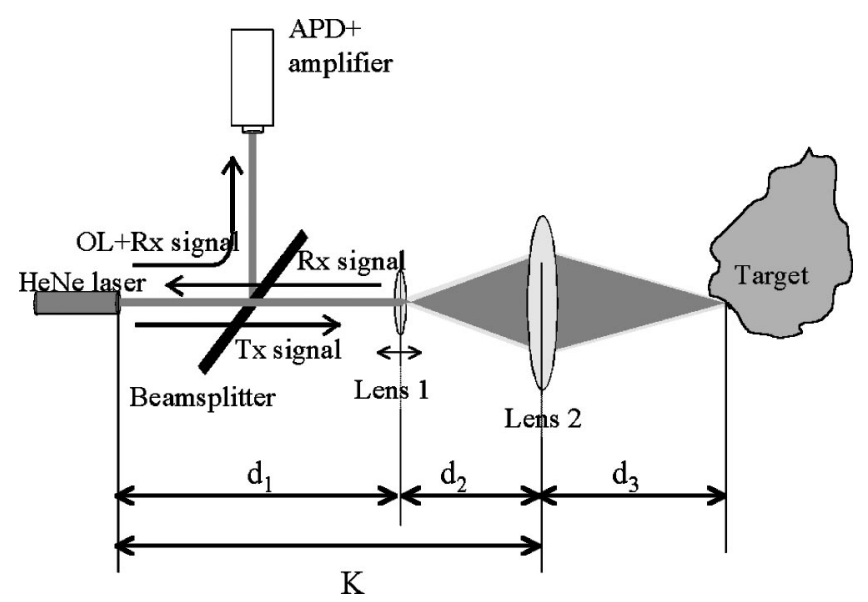

Fig. 1 Prototype layout.

This mixing mechanism is different from the modulation of the laser proposed by Rudd ${ }^{2}$ and Churnside, ${ }^{4,5}$ but was suggested by the latter in his paper. It overcomes the limitation in bandwidth for a HeNe laser interferometer described by Potter, ${ }^{3}$ because the mixing takes place outside the laser, on the active surface of the photodetector.

The prototype also presents a self-aligned configuration that makes the backpropagated local oscillator ${ }^{12}$ beam to coincide with the transmitted beam, for mixing process optimization.

\section{Optical Analysis}

\subsection{Transmitted Beam and Backpropagated Local Oscillator}

As the transmitted beam and the backpropagated local oscillator $^{12}$ beam coincide, both can be modeled by the same equations. The optical analysis presented here is based on the Gaussian beam formalism. ${ }^{13,14}$ As shown in Fig. 2, the laser output beam can be characterized by its $q$-parameter $q_{1}$, which depends on distance $z_{1}$. The output beam of the Tx/Rx/Mx optics is characterized also by its parameter $q_{3}\left(z_{3}\right)$. The value of $q_{3}$ at the output of the optics $q_{3}(0)$ can be related to the $q_{1}$ parameter at the input of the optics $q_{1}\left(d_{1}\right)$ by the following expression:

$q_{3}(0)=\frac{A q_{1}\left(d_{1}\right)+B}{C q_{1}\left(d_{1}\right)+D}$

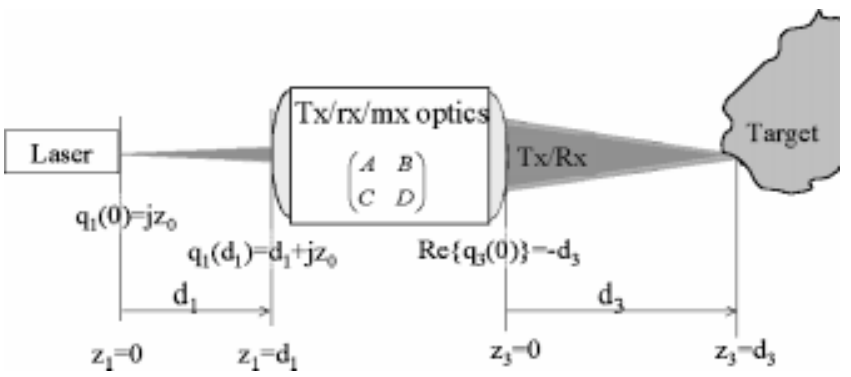

Fig. 2 Prototype layout for optical analysis. where $A, B, C$ and $D$ are the $A B C D$ parameters of the Tx/Rx/Mx optics. ${ }^{13,14}$ These parameters can be calculated in a matrix notation:

$\left[\begin{array}{cc}A & B \\ C & D\end{array}\right]=\left[\begin{array}{cc}A_{2} & B_{2} \\ C_{2} & D_{2}\end{array}\right] \times\left[\begin{array}{cc}1 & d_{2} \\ 0 & 1\end{array}\right] \times\left[\begin{array}{cc}A_{1} & B_{1} \\ C_{1} & D_{1}\end{array}\right]$,

where $d_{2}$ is the distance between the two lenses, and

$\left[\begin{array}{cc}A_{i} & B_{i} \\ C_{i} & D_{i}\end{array}\right]$

is the $A B C D$ matrix of lens $i$.

In order to achieve a good spatial coherence of the light scattered by the target, the size of the transmitted beam at its position must be minimum. The condition that the output beam has a waist at the target position $d_{3}$ is imposed as follows:

$\operatorname{Re}\left\{q_{3}(0)\right\}=-d_{3}$.

As the $D$ distance is a constant (only distance $d_{1}$ is changed) we can also add another equation:

$d_{1}+d_{2}=D$.

The result of combining Eqs. (5) and (6) is a fourth-order polynomial in $d_{1}$ as a function of $d_{3}$. The values of $d_{1}$ to have the waist of the transmitted beam at a distance $d_{3}$ of lens 2 in a range between 0 and $30 \mathrm{~m}$ vary from 8 to $24 \mathrm{~cm}$. In Figs. 3(a) and 3(b) we present two derived magnitudes of the $\mathrm{Tx} / \mathrm{Rx} / \mathrm{Mx}$ optics output beam, the spot diameter at the target surface and the convergence angle, which is used in the following study.

\subsection{Temporal Coherence Considerations: Transmitted Signal}

Two possible causes of temporal coherence loss can be identified in the laser source: the presence of multiple longitudinal modes and the spectral width of any of these modes. The presence of multiple longitudinal modes usually limits the temporal coherence in other interference based techniques (such as laser Doppler velocimetry ${ }^{1}$ or holography ${ }^{15}$ ) to approximately twice the length of the laser cavity. Nevertheless, in systems that perform a spectral analysis of the detected signal this limitation can be overcome. Figure 4 shows the resulting spectrum of the electrical signal obtained. A spectral peak appears at the Doppler frequency $\Delta f_{D}$, but some more peaks can be seen at $n \Delta f_{M} \pm k \Delta f_{D}$, where $\Delta f_{M}$ is the frequency difference between longitudinal modes, and $n$ and $k$ are positive integers.

Assuming a uniform power distribution among the $N_{M}$ different longitudinal modes in the laser, and considering that the different contributions to the peak at $\Delta f_{D}$ are uncorrelated, ${ }^{9}$ a power loss of $N_{M}$ must be considered, so Eq. (2) changes to:

$v_{\text {Dopp }}(t)=\frac{\rho_{v}}{\sqrt{N_{M}}} 2\left(\eta_{\text {het }} P_{\mathrm{LO}} P_{\mathrm{Rx}}\right)^{1 / 2} \cos \left(2 \pi \Delta f_{D} t+\chi\right)$ 


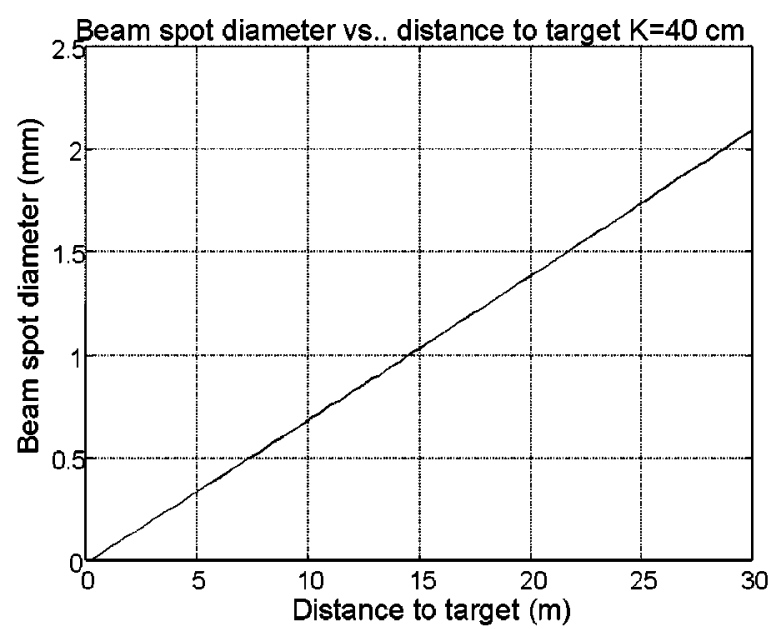

(a)

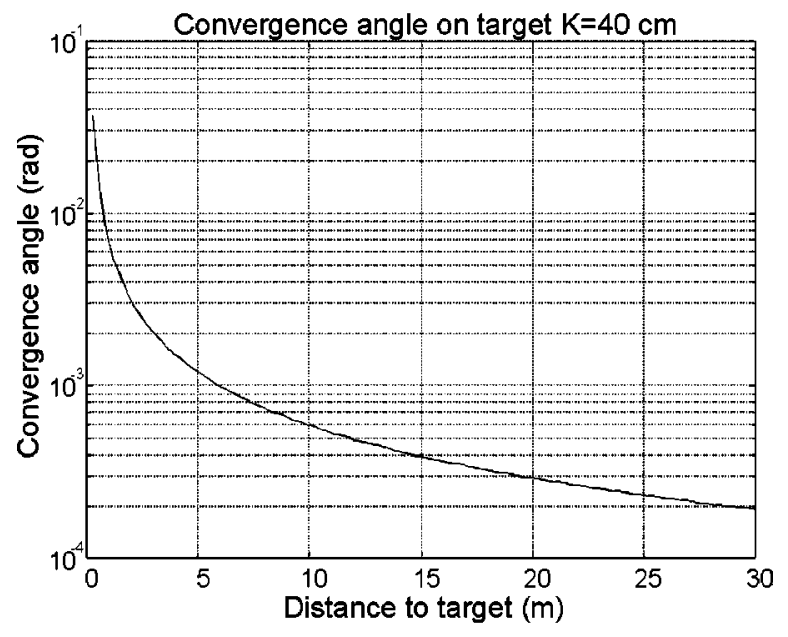

(b)

Fig. 3 (a) Diameter $2 w_{0}$ of the transmitted-beam waist as a function of distance $d_{3}$ to target on which it is assumed to lie, and (b) convergence angle of transmitted beam $2 q_{0}$ as a function of distance $d_{3}$ to the target on which the beam waist is assumed to lie.

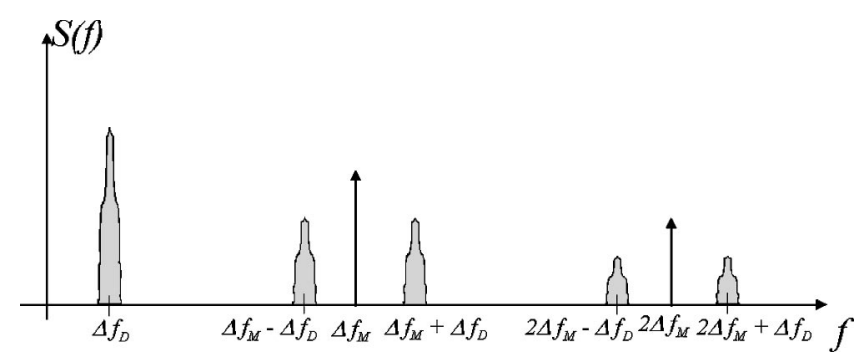

Fig. 4 Received Doppler signal spectrum.

\subsection{Temporal Coherence Considerations: Loss of Coherence of the Received Signal by Target Scattering}

The light incoherently scattered by the target is collected by the Tx/Rx/Mx optics. This light has been partially decorrelated by different mechanisms related to the scattering process. These mechanisms introduce a spectral broadening in the Doppler signal. They are identified in Figs. 5(a), 5(b) and 5(c).

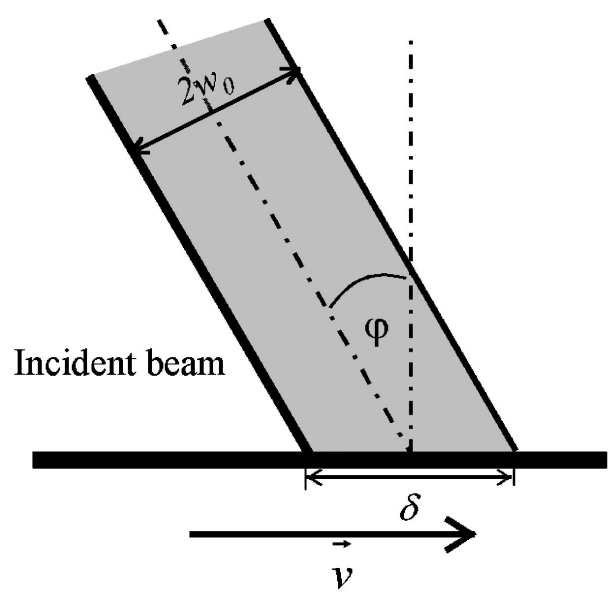

(a)

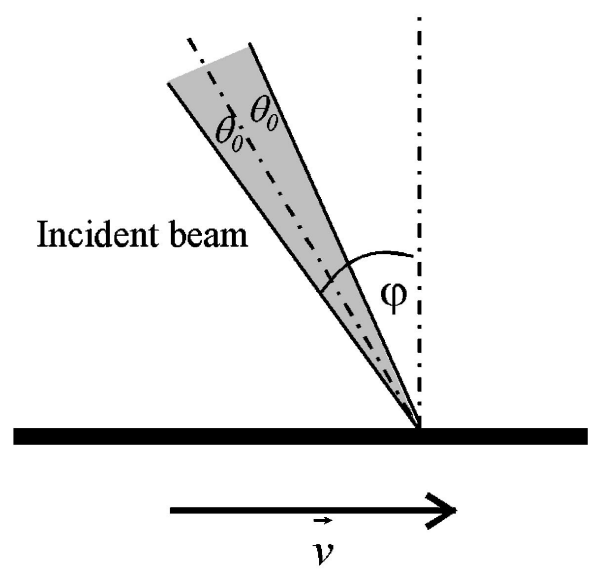

(b)

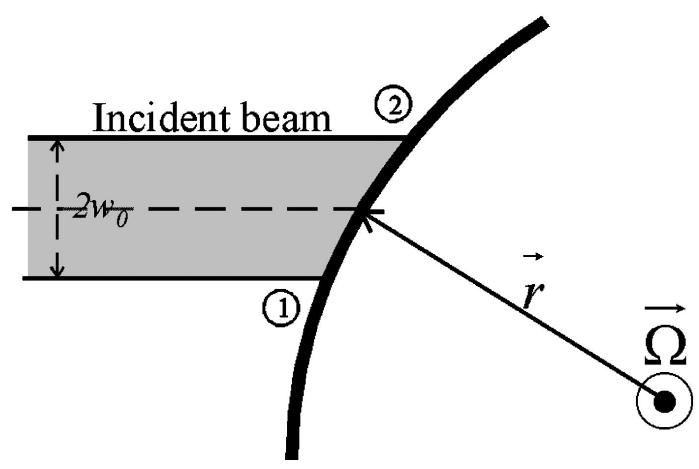

(c)

Fig. 5 (a) Spectral broadening due to loss of temporal coherence (loss due to the spatial decorrelation of the scatterers), (b) spectral broadening due to loss of temporal coherence (loss due to nonzero convergence angle), and (c) spectral broadening due to loss of temporal coherence (loss by spread of the scatterer linear velocities).

Teich $^{16}$ presented the first mechanism, loss due to the spatial decorrelation of the scatterers present on the target surface, and it is sketched in Fig. 5(a). According to Fig. 5 (a), the maximum coherence time $\tau_{c}$ of the light scattered by the target is:

$\tau_{c} \approx \frac{\delta}{v}$ 
where $\delta$ is the diameter of the laser spot on the target, and $v$ is the target lineal speed.

Considering that $\delta=2 w_{0} / \cos \varphi$, the spectral broadening $\Delta f_{s}$ can be calculated as:

$\Delta f_{s}=\frac{1}{\tau_{c}} \approx \frac{v \cos \varphi}{2 w_{0}}$,

where $v$ is the linear speed of the target in the incidence point, $\varphi$ is the nominal incidence angle, and $w_{0}$ is the incident beam waist radius at $1 / e^{2}$ of its maximum illumination value.

A second spectral broadening mechanism is due to the convergence angle of the transmitted beam, which makes the value of the incident angle $\varphi$ not unique. This situation is presented in Fig. 5(b). The corresponding spectral broadening $\Delta f_{\theta_{0}}$, due to the incident angle range, can be written ${ }^{9}$ as:

$\Delta f_{\theta_{0}}=\frac{4 v}{\lambda} \cos \varphi \sin \theta_{0} \approx \frac{4 v}{\lambda} \theta_{0} \cos \varphi \approx \frac{4 v}{\pi w_{0}} \cos \varphi$,

where $\lambda$ is the laser wavelength, and $\theta_{0}$ is the beam convergence half angle that must be very small for the paraxial approximation applied to be valid. ${ }^{13,14}$

The third mechanism appears only when the illuminated spot on the target rotates with instantaneous angular speed $W$ and radius vector $\mathbf{r}$. The nonnegligible size of the transmitted beam waist on the target encompasses a range of values of the longitudinal component of linear velocity, as presented in Fig. 5(c); which produces a spectral broadening in the Doppler signal. This rotating-target broadening $\Delta f_{r}$ can be calculated ${ }^{9}$ as:

$\Delta f_{r}=\frac{4 \Omega w_{0}}{\lambda}$.

The three effects can be combined in a root mean square (rms) manner to obtain an overall spectral broadening ${ }^{9}$ $\Delta f_{\text {rot }}$ :

$$
\begin{aligned}
\Delta f_{\mathrm{rot}} & =\left(\Delta f_{s}^{2}+\Delta f_{\theta_{0}}^{2}+\Delta f_{r}^{2}\right)^{1 / 2} \\
& =\left[\frac{\cos ^{2} \varphi\left(\pi^{2}+64\right)}{4 \pi^{2} w_{0}^{2}} v^{2}+\frac{16 w_{0}^{2}}{\lambda^{2}} \boldsymbol{\Omega}^{2}\right]^{1 / 2} .
\end{aligned}
$$

\subsection{Considerations on the Spatial Coherence of the Received Signal}

To mix efficiently with the local oscillator the received signal must show spatial coherence over a significant part of the receiving aperture. Due to the roughness of the target surface (of the order of the carrier wavelength), the scattering must be considered as spatially incoherent.

Nevertheless, the Van Cittert-Zernike theorem ${ }^{17}$ predicts a gain in coherence due to propagation. Let us consider the situation described in Fig. 6, in which the lidar system focuses the transmitted beam on the target surface. The illuminated spot on the target shows an intensity Gaussian distribution of radius $w_{0}$ at $1 / e^{2}$ from the maximum:

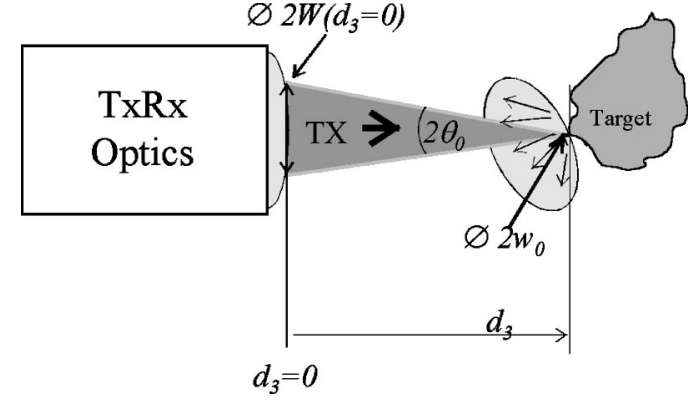

Fig. 6 Illustration for the computation of the coherence area of the received signal.

$I(r)=I_{0} \exp \left[\left(2 r^{2} / w_{0}^{2}\right)\right]$,

where $I_{0}$ is the maximum intensity and $r$ is the distance to the center of the spot.

According to the Van Cittert-Zernike theorem, the modulus of the complex coherence factor at a distance $z$ from the target can be calculated ${ }^{9}$ as:

$\left|\mu_{12}(\rho)\right|=\exp \left\{-\left[\frac{w_{0}^{2}}{8(\lambda z)^{2}} \rho^{2}\right]\right\}$.

This function falls to $1 / e^{2}$ for a value $\rho=\rho_{c}=4(\lambda z) / w_{0}$. We call $\rho_{c}$ the coherence radius of the backscattered light. It can be compared to the radius of the transmitted beam at the $\mathrm{Tx} / \mathrm{Rx} / \mathrm{Mx}$ optics output, $W_{3}(0)$ :

$W_{3}(0)=\frac{\lambda z}{\pi w_{0}}$.

Thus, the coherence radius of the backscattered light at the optics output is $4 \pi$ times greater than the transmitted beam radius. According to Ref. 11, we calculate the average receiving area as:

$\left\langle A_{R}\right\rangle=\left(\left\{\pi\left[W_{3}(0)\right]^{2}\right\}^{-1}+\left(\pi \rho_{c}^{2}\right)^{-1}\right)^{-1}$.

Thus, in a focused system, according to the previous results concluding that $\rho_{c} \gg W_{3}(0)$, the receiving area is nearly equal to the transmitted beam section area at the Tx/Rx/Mx optics output. This means that we can assume very little loss due to loss of spatial coherence.

Atmospheric turbulence must be considered as an additional mechanism of reducing effective receiving area. According to Hufnagel ${ }^{18}$ for propagation through a homogeneously turbulent atmosphere the atmospheric coherence diameter $r_{0}$ can be calculated, for a spherical wave:

$r_{0}=0.332\left(\frac{\lambda^{2}}{C_{n}^{2} R}\right)^{3 / 5}$, 
where $C_{n}^{2}$ is the so-called structure constant of the index of refraction, ${ }^{18}$ and $R$ is the propagation length. For a standard turbulence in open space, much stronger than that expected in a laboratory environment, we can consider $C_{n}^{2}$ $\approx 10^{-15} \mathrm{~m}^{-2 / 3}$. For a propagation length $R \sim 20 \mathrm{~m}, r_{0}$ is greater than $1 \mathrm{~m}$, much greater than any aperture considered on the system, specially the effective receiving aperture $\left\langle A_{R}\right\rangle$, whose diameter is around $1 \mathrm{~cm}$. Thus, no reduction due to atmospheric turbulence is considered.

\section{Power Budget and Tolerance in the Distance Adjustment}

\subsection{Signal and Noise Power Budget}

According to Eq. (7), the average power of the Doppler signal depends on the voltage responsivity of the photoreceiver $\rho_{v}$ the number of longitudinal modes present in the laser light $N_{M}$, the power of the local oscillator beam $P_{\text {LO }}$ and the average received light power $\left\langle P_{\mathrm{Rx}}\right\rangle$. In our experimental setup we used a beamsplitter with two reflecting sides, characterized by an average reflection coefficient $r_{\mathrm{BS}}=0.05$. So the local oscillator power can be calculated as:

$P_{\mathrm{LO}}=r_{\mathrm{BS}} P_{T} L_{D}$,

where $P_{T}$ is the optical power transmitted by the laser, and $L_{D}$ is the power loss due to spilling of the local oscillator power over the APD active area when the latter is smaller than the local oscillator beam cross section. This loss can be calculated as:

$L_{D}=\frac{d_{\mathrm{APD}}^{2}}{d_{\mathrm{LASER}}^{2}}$,

where $d_{\mathrm{APD}}$ is the APD diameter and $d_{\text {LASER }}$ the local oscillator beam diameter, equal to that of the laser output beam.

The average power $\left\langle P_{\mathrm{Rx}}\right\rangle$ of light backscattered by the target collected by the $\mathrm{Tx} / \mathrm{Rx} / \mathrm{Mx}$ optics and directed onto the active surface can be calculated through the expression:

$\left\langle P_{\mathrm{Rx}}\right\rangle=\left\langle A_{R}\right\rangle L_{D}\left(1-2 r_{\mathrm{BS}}\right)^{2} r_{\mathrm{BS}} \frac{\sigma^{0} P_{T}}{\pi R^{2}}$,

where $\sigma^{0}$ is the backscattering coefficient of the surface of the target and $R$ is the distance to the target.

Expression (7) also includes a heterodyne efficiency term $\eta_{\text {het }}$. According to the earlier considerations, and based on the definition by Rye and Frehlich, ${ }^{11}$ this term is calculated as

$\eta_{\text {het }}=\frac{L_{D}^{2}\left\langle A_{R}\right\rangle L_{\mathrm{pol}}}{A_{\mathrm{RL}}}$,

where $A_{\mathrm{RL}}$ is the geometrical area of the receiving aperture, and $L_{\mathrm{pol}}$ is the loss due to the depolarization of the backscattered light (not considered in Ref. 11); adopting a pessimistic criterion, we will consider $L_{\mathrm{pol}}=0.5$. With this definition $\eta_{\text {het }}$ varies with the distance to the target, with a typical value of $\eta_{\text {het }}=0.4 \%$ being obtained. The main reason for this low value is the fact that the lens diameter is much larger than the BPLO diameter at the lens plane, which was deliberately chosen to avoid the truncation of the transmitted beam. This low value for $\eta_{\text {het }}$ is compensated in the total power budget by an accordingly increased value of the received power $P_{\mathrm{Rx}}$, according to Eq. (7).

We can consider that our photoreceiver works in a shotnoise limited regime, due to the high value of light arriving to the APD active surface. So we can calculate the rms noise voltage $\left\langle v_{n}^{2}\right\rangle$ at the photoreceiver output through the expression $^{19}$ :

$\left\langle v_{n}^{2}\right\rangle=2 e G_{\mathrm{APD}} F G_{Z} \rho_{v} P_{\mathrm{LO}} \mathrm{BW}$,

where $e$ is the electron charge, $G_{\mathrm{APD}}$ is the avalanche gain of the APD, $F$ is the excess noise factor of the APD, $G_{Z}$ is the transimpedance gain of the photoreceiver electronics, and $\mathrm{BW}$ is the electric bandwidth of the photoreceiver.

\subsection{Criterion on Tolerance in the Distance Adjustment}

The system presented employs a beam focused onto the target surface, as shown in Fig. 6. If the distance to target is known within a certain tolerance, the performance of optical mixing can be reduced. In this subsection, we present a criterion $^{9}$ that enables us to calculate a maximum tolerance in distance for every situation.

According to the Van Cittert-Zernike theorem, the coherence area of the light backscattered by an incoherent surface with Gaussian distributed illumination $A_{\text {coh }}$ (see Subsection 3.4) is given by

$A_{\mathrm{coh}}\left(w_{0}, d_{3}\right)=\pi \rho_{c}^{2}=16 \pi\left(\frac{\lambda d_{3}}{w_{0}}\right)^{2}$.

For our system, the value of $w_{0}$ is related to the characteristics of the transmitted Gaussian beam, and is given by:

$W_{3}\left(d_{3}, \Delta z\right)=w_{03}\left(d_{3}\right)\left\{1+\left[\frac{\Delta z}{z_{03}\left(d_{3}\right)}\right]^{2}\right\}^{1 / 2}$,

where $\Delta z$ is the distance from the beam waist, $W_{3}\left(d_{3}, \Delta z\right)$ is the radius of the transmitted beam at $1 / e^{2}$ of the maximum intensity, $w_{03}\left(d_{3}\right)$ is the transmitted beam waist radius for a beam focused at a distance $d_{3}$, and $z_{03}\left(d_{3}\right)$ is the Rayleigh distance of the transmitted beam, which is the imaginary part of the $q_{3}$ parameter. The parameters $z_{03}$ and $w_{03}$ are related by the expression:

$z_{03}\left(d_{3}\right)=\frac{\pi w_{03}^{2}\left(d_{3}\right)}{\lambda}$.

According to the Van Cittert-Zernike ${ }^{17}$ theorem, as the distance $\Delta z$ grows, the target gets out of focus and the size of the light spot increases, which makes the coherence area smaller at a given distance of the scattering spot. A loss of detected signal can be associated to this loss in spatial coherence by means of the reduction of effective receiving 
Rodriguez, Comeron, and Garcia: Homodyne laser radar system ...

area. To assess a maximum tolerance in the distance adjustment a loss in the detected Doppler signal equal to the loss in coherence area is assumed. The criterion of maximum tolerance in distance considers that the system must work with a minimum signal to noise ratio $\mathrm{SNR}=10 \mathrm{~dB}$, and can be written in the following way:

$\frac{A_{\mathrm{coh}}\left(\Delta z=0, d_{3}\right)}{A_{\mathrm{coh}}\left(\Delta z, d_{3}\right)} \leqslant \frac{\operatorname{SNR}\left(d_{3}\right)}{10}$,

where $\operatorname{SNR}\left(d_{3}\right)$ is the SNR for a target that is situated at a known distance $d_{3}$ when the transmitted beam is perfectly focused on its surface.

According to this criterion, the maximum tolerance in distance $\left|\Delta z_{\text {tol }}\right|$ for such a system can be calculated with the following expression ${ }^{9}$

$\left|\Delta z_{\text {tol }}\right| \leqslant z_{03}\left(d_{3}\right)\left[\frac{\operatorname{SNR}\left(d_{3}\right)}{10}-1\right]^{1 / 2}$,

which will be valid only for those values of $d_{3}$ that allow a $\operatorname{SNR}\left(d_{3}\right)$ equal to or greater than $10 \mathrm{~dB}$.

Of course, this is a pessimistic criterion because it considers that a reduction of the coherence area of the backscattered light results in a proportional reduction of Doppler signal. Nevertheless, it has proven to be very simple and describes quite reasonably the actual behavior of our experimental prototype.

\section{Experimental Results}

To test the homodyne coherent laser radar system, a prototype has been built and tested. The following elements were used:

1. a Siemens model LGK 7627 laser transmitter with a wavelength of $632.8 \mathrm{~nm}$, a typical output power of 10 $\mathrm{mW}$, a spatial mode of $\mathrm{TEM}_{00}$, a beam size at $1 / e^{2}$ intensity of $0.8 \mathrm{~mm}$, and a longitudinal beam spacing of $438 \mathrm{MHz}$. (Number of longitudinal modes was estimated to be 4.)

2. an Analog Modules model 713-4 photoreceiver with a EG\&G C30902E photodiode, a nominal responsivity at $632.8 \mathrm{~nm}$ of $60 \mathrm{~A} / \mathrm{W}$, a responsivity at 632.8 $\mathrm{nm}$ and $125 \mathrm{~V}$ of $7 \mathrm{~A} / \mathrm{W}$, and active area diameter of $0.5 \mathrm{~mm}$, an electrical bandwidth of 200 to $250 \mathrm{MHz}$, and a transimpedance gain of $20 \mathrm{k} \Omega$.

The APD included in the photoreceiver has been biased at $\sim 125 \mathrm{~V}$, thus reducing the avalanche gain, instead of its nominal value $230 \mathrm{~V}$ due to the high local oscillator power level. In fact, at its nominal bias, the high avalanche gain made that the C30902E could not dissipate the heat produced by the dc current induced by the local oscillator.

The value of the rest of the parameters are as follows:

Distance $D=40 \mathrm{~cm}$,

Lens 1: focal length $=1 \mathrm{~cm}$, diameter $=0.9 \mathrm{~cm}$,

Lens 2: focal length $=15 \mathrm{~cm}$, diameter $=5 \mathrm{~cm}$.

The laboratory target was a $25-\mathrm{cm}$-radius rotating disk made of PVC. Its cylindrical surface was enhanced using an aluminum plate covered in paper. We performed some measurements on the backscattering characteristics of the paper employed, which showed that it can be considered as a Lambertian scatterer, with a backscattering coefficient $\sigma^{0}=0.775$.

The Doppler signal was observed in a general-purpose spectrum analyzer with a resolution bandwidth RBW $=100 \mathrm{kHz}$. This adjustment proved to be the most adequate for the obtained electrical signal. Nevertheless, due to the spectrum broadening effects presented in Subsection 3.4, the spectral width of the observed signal is larger than the indicated RBW, and so the height of the spectral peak observed in the spectrum analyzer is slightly diminished. This reduction was considered in the calculations presented in Figs. 7(a) and 8(a).

Two series of measurements were performed, the first one with the disk rotating at approximately $400 \mathrm{rpm}$ and the second one with an angular velocity of approximately 1300 rpm. In both series, we measured the Doppler signal power, its spectral width, and the noise level at the photoreceiver output. In the low-speed series, we also measured the tolerance in the distance adjustment of the system.

When the target rotates at $400 \mathrm{rpm}$, the detected Doppler shift is $16 \mathrm{MHz}$ for $\varphi=30 \mathrm{deg}, 23 \mathrm{MHz}$ for $\varphi=45 \mathrm{deg}$ and $29 \mathrm{MHz}$ for $\varphi=60 \mathrm{deg}$, which coincide with the expected values. Figure 7 (a) shows the Doppler signal power and noise versus distance for the three different values of the incidence angle $\varphi$; the Doppler signal power measurements show power values slightly lower than the calculations. The maximum working distance, defined with a criterion of a minimum $\mathrm{SNR}=10 \mathrm{~dB}$, when the spectrum analyser resolution bandwidth is $\mathrm{RBW}=100 \mathrm{kHz}$, is $16 \mathrm{~m}$ for $\varphi=30$ and $45 \mathrm{deg}$ and $12 \mathrm{~m}$ for $\varphi=60 \mathrm{deg}$.

Figure 7(b) shows the Doppler signal spectral width at $-10 \mathrm{~dB}$. The election of this definition of spectral width was made for practical measurement reasons (specifically, good signal shape visibility) and because the theoretical calculations were made on a $1 / e^{2}$ basis, which is approximately $-8.7 \mathrm{~dB}$. Good agreement can be found between practice and theory at distances greater than $2 \mathrm{~m}$.

Figure 7(c) shows the measurements of tolerance in distance. Most of the measurements are better than theory, probably because of the pessimistic criterion used, and a general tendency of an initial growing, followed by a quick fall, predicted by the theory (continuous curves), can be observed for the three series of measurements $(\varphi=30,45$ and $60 \mathrm{deg}$ ). Some points, however, especially at the incidence angle of $30 \mathrm{deg}$, depart from the general trend likely due to problems in the target positioning. According to a criterion of nonzero tolerance in distance, the maximum working distance can be considered as $\sim 16 \mathrm{~m}$ for $\varphi=30$ and $45 \mathrm{deg}$ and $14 \mathrm{~m}$ for $\varphi=60 \mathrm{deg}$.

Figures 8(a) and 8(b) show, respectively, the Doppler signal power and spectral width for the target rotating at an angular speed of approximately $1300 \mathrm{rpm}$. The corresponding Doppler shifts measured are $54 \mathrm{MHz}$ for $\varphi=30 \mathrm{deg}, 76$ $\mathrm{MHz}$ for $\varphi=45 \mathrm{deg}$ and $93 \mathrm{MHz}$ for $\varphi=60 \mathrm{deg}$, corresponding with the theoretical values. Note that the signal power peak [see Fig. 8(a)] observed in the spectrum analyzer experiments experienced a slight fall due to the extra spectral widening, predicted by Eq. (12). Due to this visibility reduction, the maximum working range falls to approximately $12 \mathrm{~m}$. 


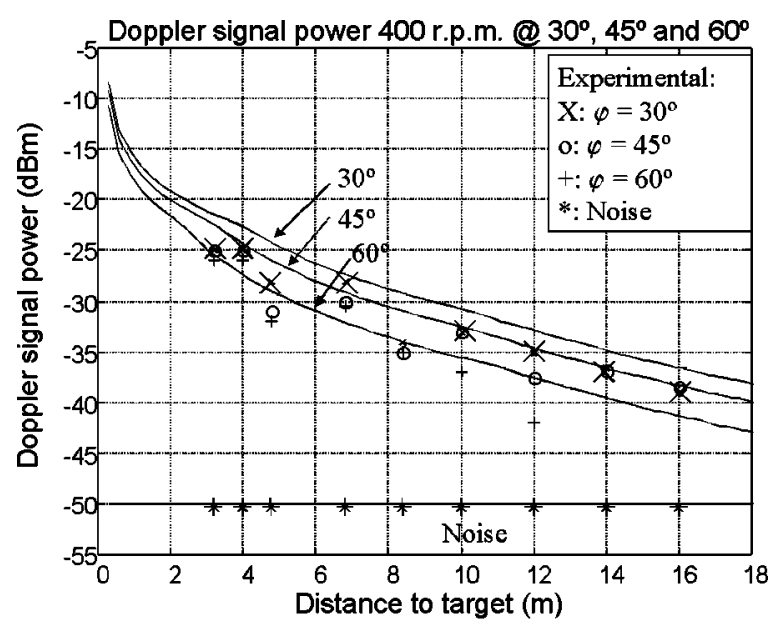

(a)

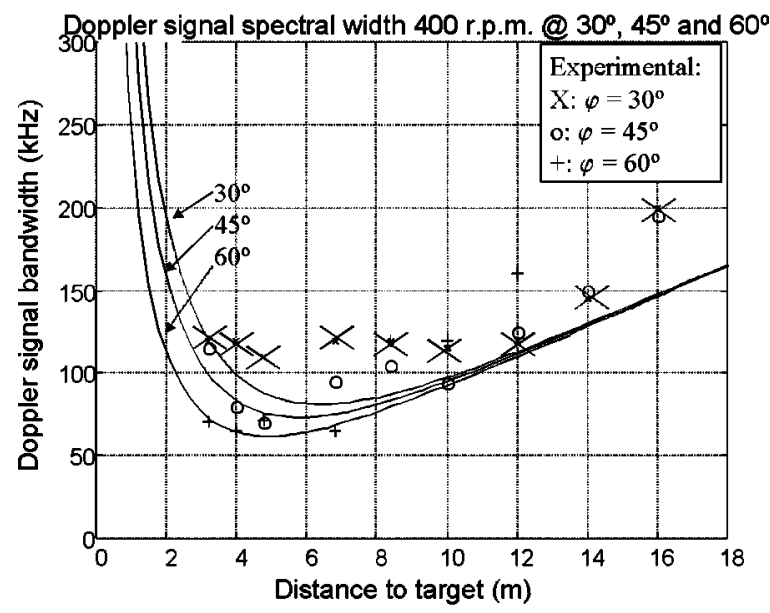

(b)

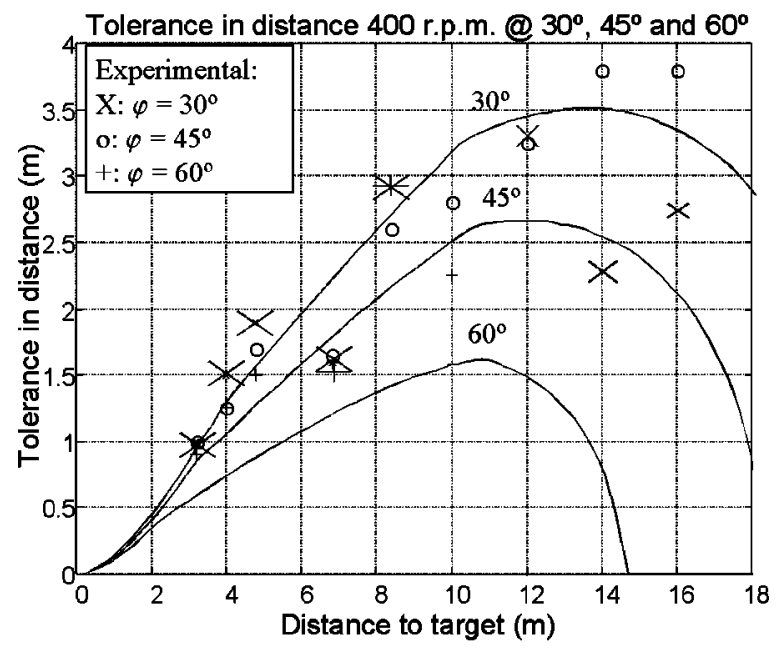

(c)

Fig. 7 (a) Calculated (continuous lines) and measured (symbols) (a) Doppler signal power, (b) Doppler signal bandwidth, and (c) tolerance in the distance adjustment of the lidar as a function of distance and for three different incidence angles $\varphi$ for the target rotating at $400 \mathrm{rpm}$.

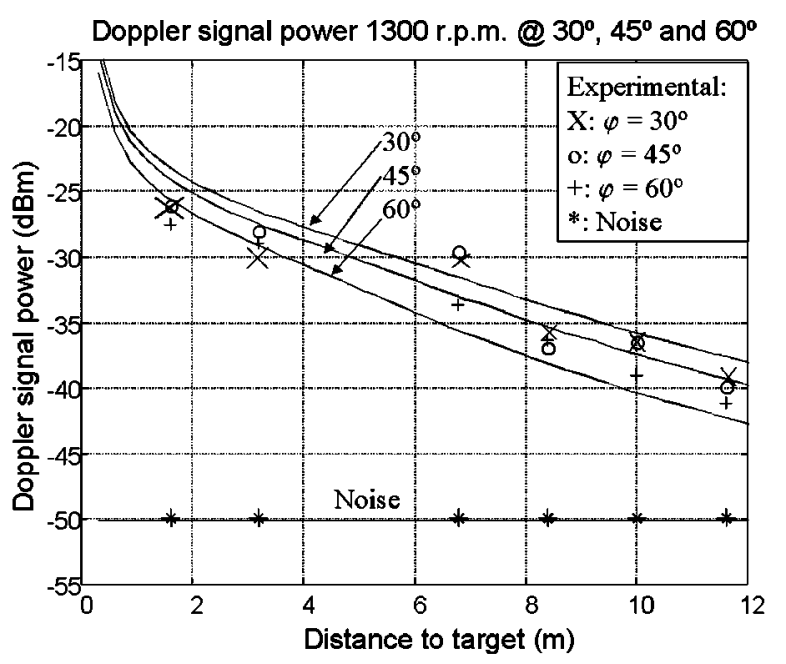

(a)

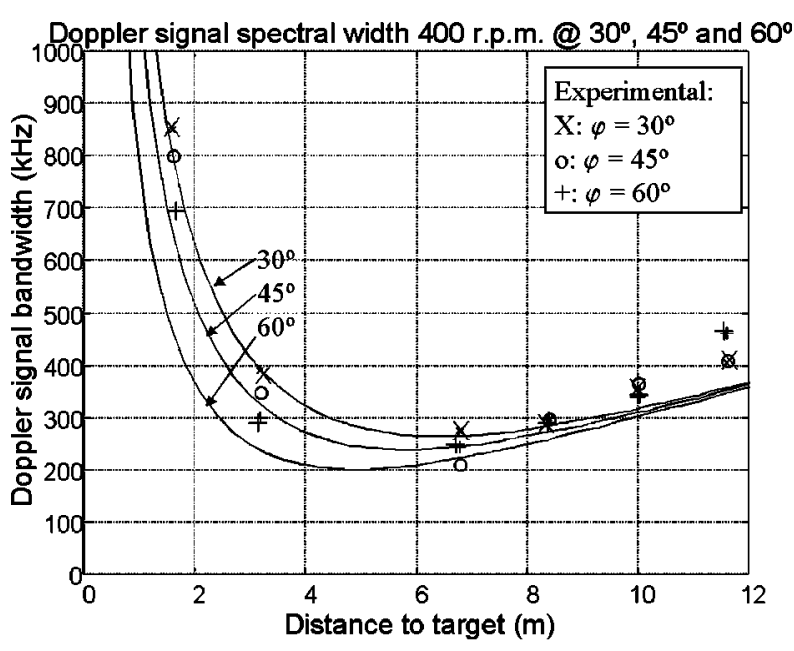

(b)

Fig. 8 Calculated (continuous lines) and measured (symbols) (a) Doppler signal power and (b) Doppler signal bandwidth as a function of distance and for three different incidence angles $\varphi$ for the target rotating at $1300 \mathrm{rpm}$.

The measured spectral widths [see Fig. 8(b)] correlate acceptably to the calculated values.

\section{Conclusions}

A very simple system that enables us to perform Doppler measurements of the velocity of hard targets was presented. Few optical elements, common nonexpensive electro-optic components and auto aligning are its main advantages. An optical analysis that includes computations regarding the characteristics of the different beams and the coherence between the different signals was performed. Also a power budget was calculated. A criterion for calculating the tolerance in the distance adjustment of the system was pro- 
posed. Finally, experimental results obtained with a laboratory rotating target were presented. The experimental results show the capability of the system to perform Dopplershift measurements at distances up to $16 \mathrm{~m}$.

\section{Acknowledgments}

This work was supported by the Spanish Government through grants CICYT TIC 431-93 and AMB96-1144-C0202 .

\section{References}

1. L. E. Drain, The Laser Doppler Technique, Wiley, Norwich, Great Britain (1980).

2. M. J. Rudd, "A laser Doppler velocimeter employing the laser as a mixer-oscillator," J. Sci. Instrum. 1 (Ser. 2), 723-726 (1968).

3. I. C. Potter, "Frequency response of the 6328- $\AA$ helium-neon laser interferometer," J. Appl. Phys. 40(12), 4770-4776 (1969)

4. J. H. Churnside, "Laser Doppler velocimetry by modulating a $\mathrm{CO}_{2}$ laser with backscattered light," Appl. Opt. 23(1), 61-66 (1984).

5. J. H. Churnside, "Signal-to-noise in a backscattered-modulated Doppler velocimeter," Appl. Opt. 23(13), 2097-2106 (1984).

6. S. Shinohara et al., "Laser Doppler velocimeter using the self-mixing effect of a semiconductor laser diode," Appl. Opt. 25(9), 1417-1419 (1986).

7. M. H. Koelnik et al., "Laser Doppler velocimeter based on the selfmixing effect in a fiber-coupled semiconductor laser: theory," Appl. Opt. 31(18), 3401-3408 (1992).

8. A. Rodríguez, A. Comeron et al., "Sistema lidar coherente monoestático de baja potencia para medida de velocidad lineal y de rotación de blancos sólidos," Patent No. 9702070, Oficina Española de Patentes y Marcas (1997).

9. A. Rodríguez, "Sistemas lidar coherentes e incoherentes de baja potencia para la detección de velocidad de blancos sólidos," $\mathrm{PhD}$ Thesis disertation, Universitat Politécnica de Catalunya (Dec. 1998).

10. B. J. Rye, "Antenna parameters for incoherent backscatter heterodyne lidar,"' Appl. Opt. 18(9), 1390-1398 (1979).

11. B. J. Rye and R. G. Frehlich, "Optimal truncation and optical efficiency of an apertured coherent lidar focused on an incoherent backscatter target," Appl. Opt. 31(15), 2891-2899 (1992).

12. A. E. Siegman, "The antenna properties of optical heterodyne receivers," Proc. IEEE 54(10), 1350-1356 (1966).

13. A. Yariv, Quantum Electronics, 3rd ed., Wiley, New York (1989).

14. H. Kogelnik and T. Li, "Laser beams and resonators," Appl. Opt. 5(10), 1550-1567 (1966).

15. N. Abramson, The Making and Evaluation of Holograms, Academic Press (1981)

16. M. C. Teich, "Infrared heterodyne detection," Proc. IEEE 56(1), 37-46 (1968).

17. J. W. Goodman, Statistical Optics, Wiley, New York (1985).
18. R. E. Hufnagel, "Propagation through atmospheric turbulence," Chap. 6 in The Infrared Handbook, William L. Wolfe and George J. Zissis, Eds., Office of Naval Research, Dept. of the Navy, Washington, DC (1978).

19. J. M. Senior, Optical Fiber Communications: Principles and Practice, Prentice-Hall, Englewood Cliffs, NJ (1985).

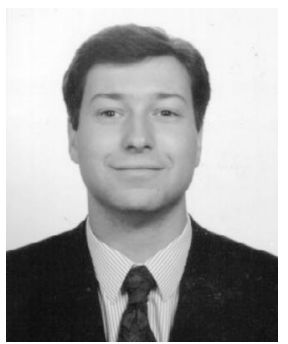

Alejandro Rodriguez received his telecommunication engineer degree from the Technical University of Madrid (UPM) in 1993 and his $\mathrm{PhD}$ in telecommunication engineering from the Technical University of Catalonia (UPC) in 1998. Since 1995 he has been an associate professor with the Department of Signal Theory and Communications, UPC. His research interests include low power coherent and incoherent laser radar systems and atmospheric lidar systems. Dr. Rodriguez is a member of SPIE.

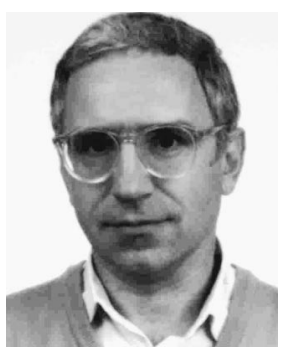

Adolfo Comeron received his telecommunication engineer degree from the Telecommunication Engineer School of Barcelona in 1976 and his DEA and Drlng degrees from the Paris-XI University, Orsay, in 1977 and 1980, respectively. He is currently professor with the Technical University of Catalonia, Barcelona, Spain. His research activities have included the study of nonlinear devices at IR wavelengths and the development of microwave and millimeter-wave receivers for satellite communication systems, and he is currently focused on free-space optical communications and remote detection and sensing at optical wavelengths. Dr. Comeron is a member of SPIE.

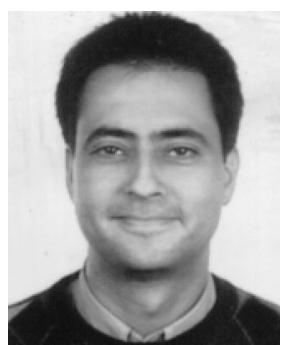

David Garcia received a degree in telecommunications in 1993 and the telecommunication engineering degree in 1998, both from the Technical University of Catalonia (UPC). Since 1996 he has been an associate professor with the Department o Signal Theory and Communications (UPC). His research interests include low power coherent laser radar and laser Doppler anemometry systems. 